Ophthalmologe $2021 \cdot 118: 439-448$

https://doi.org/10.1007/s00347-021-01362-z

Angenommen: 23. Februar 2021

Online publiziert: 17. März 2021

(c) Springer Medizin Verlag GmbH, ein Teil von Springer Nature 2021

\section{Redaktion}

C. Erb

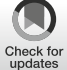

Die neuroprotektiven therapeutischen Ansätze bei der Glaukomerkrankung haben sich bisher in den klinischen Studien nicht bewährt. Dabei zielten die neuroprotektiven Strategien bisher im Wesentlichen auf Netzhaut und Sehnerv. In den letzten Jahren gewonnene Erkenntnisse zur neuronalen Degeneration im Verlauf der gesamten Sehbahn und des Gehirns bei der Glaukomerkrankung eröffnen neue Möglichkeiten für neuroprotektive Therapien.

Die Glaukomerkrankung ist weltweit die zweithäufigste Ursache für Blindheit. Die epidemiologischen Analysen der WHO weisen Glaukom als die zweithäufigste Ursache irreversibler Erblindung aus, verantwortlich für $12 \%$ der Erblindungsfälle [54]. Zurzeit Sind weltweit etwa 70 Mio. Menschen an Offenwinkelglaukomen erkrankt, etwa 7 Mio. Menschen sind beidseits infolge der glaukomatösen Optikusatrophie erblindet.

\section{》) Infolge des symptomfreien Beginns sind nur etwa $50 \%$ aller Glaukome derzeit diagnostiziert}

Infolge des symptomfreien Beginns sind nur etwa $50 \%$ aller Glaukome derzeit diagnostiziert. Die multifaktorielle Ätiologie dieser neurodegenerativen Erkrankung ist bis heute nicht im Detail geklärt. Als derzeit einziger evidenzbasierter modifizierbarer Risikofaktor für die Entstehung sowie die Progression der glaukomatösen Optikusatrophie steht die medikamentöse und chirurgische Senkung

Anselm G. M. Jünemann' $\cdot$ P. Grieb ${ }^{2} \cdot$ R. Rejdak ${ }^{1,2}$

'Department of General and Pediatric Ophthalmology Service, Medical University of Lublin, Lublin, Polen ${ }^{2}$ Department of Experimental Pharmacology, Mossakowski Medical Research Centre, Polish Academy of Sciences, Warschau, Polen

\title{
Bedeutung von Citicolin bei der Glaukomerkrankung
}

des intraokularen Drucks (IOD) im Zentrum des therapeutischen Glaukom-Managements. Da die Senkung des Augeninnendrucks einen positiven Effekt auf die Glaukomerkrankung hat [23], wird diese von der World Health Organization (WHO) als eine vermeidbare Ursache für Erblindung angesehen und insofern als „priority eye disease“ gelistet.

\section{Stellenwert der Neuroprotektion}

Für die Neuroprotektion ist es ein weiter Weg von der Grundlagenforschung in die Klinik. Das weite Feld der Neuroprotektion im Rahmen der Glaukomerkrankung zeigt mit der Entwicklung von neurotrophen, antioxidativen, antiexzitotoxischen, antiischämischen, antiinflammatorischen, antiapoptotischen und immunmodulatorischen Therapieansätzen vielversprechende Fortschritte, die Neurodegeneration zu vermindern und somit die Sehfunktion zu stabilisieren.

Jedoch konnten sich trotz eindrucksvoller Ergebnisse experimenteller Studien die zahlreichen getesteten Substanzen i. d. R. in den klinischen Studien nicht bewähren. Eine mögliche Ursache hierfür liegt in der inadäquaten Wahl funktioneller Endpunkte in den klinischen Studien. Ein hierfür bekanntes Beispiel in der Glaukomtherapie ist Memantin, für das trotz nachgewiesener Neuroprotektion in tierexperimentellen Studien [4] in 2 randomisierten doppelmaskierten placebokontrollierten multizentrischen Studien über 48 Monate kein protektiver Effekt im Hinblick auf die Gesichtsfeldprogression aufgezeigt werden konnte [50]. Auch wenn dieses Studienergebnis einen ne- gativen Einfluss auf die Investitionen in neuroprotektive Therapeutika durch die pharmazeutische Industrie zur Folge hatte, wurden dennoch seit der Memantinstudie im Jahr 2008 protektive Strategien für die Glaukomerkrankung weiterentwickelt.

\section{》) Die glaukomatöse Neurodegeneration umfasst die gesamte Sehbahn}

Dabei konzentrierte sich die Neuroprotektion bei der Glaukomerkrankung bisher im Wesentlichen auf Netzhaut und Sehnerv. In den letzten Jahren wurde jedoch in vielen experimentellen und klinischen Studien gezeigt, dass bei der Glaukomerkrankung die neuronale Degeneration nicht nur auf der Höhe von Netzhaut und Sehnerv, sondern im Verlauf der gesamten Sehbahn und des Gehirns erfolgt. Die glaukomatöse Neurodegeneration umfasst also die gesamte Sehbahn.

Die neuroanatomischen Verhältnisse machen die Beteiligung der zerebralen Strukturen an der glaukomatösen Neurodegeneration verständlich: Der Sehnerv und seine Glia, bestehend aus Oligodendrozyten und Astrozyten, ist als Teil des zentralen Nervensystems innerhalb der Blut-Hirn-Schranke gelegen und von Meningen umgeben. Die Axone der retinalen Ganglienzellen bilden nach Durchtritt durch die Lamina cribrosa den intraorbitalen, intrakanalikulären und intrakranialen Sehnerv, das Chiasma und den Tractus opticus. Zwischen dem Sehnervenkopf als Beginn des eigentlichen Sehnervs und der Sehrinde (Area 17) befindet sich nur eine 
Synapse, sodass eine transsynaptische oder transneuronale Degeneration entlang dieser einzelnen Synapse sowohl zu einem primären pathologischen Prozess am Sehnervenkopf als auch zu Veränderungen im visuellen Kortex führt. Dementsprechend sind sekundäre Veränderungen der zentralen Sehbahn und anderer Hirnregionen nach einem Verlust an retinalen Ganglienzellen (RGZ) in Form unterschiedlicher Pathologien beschrieben. Auch die Neurodegeneration bei der Glaukomerkrankung ist nicht nur auf die Netzhaut, hier v. a. auf die Schicht der retinalen Ganglienzellen, und den Sehnerv begrenzt, sondern breitet sich entlang der gesamten retinogenikulokortikalen Sehbahn bis zum visuellen Kortex aus [28].

So weisen funktionelle Veränderungen insbesondere in den visuell evozierten Potenzialen (VEP) [18] auf eine supraretinale Schädigung hin.

Auch mittels Neuroimaging konnten die Veränderungen in der posterioren Sehbahn nachgewiesen werden. Mehrere Fall-Kontroll-Studien zeigten, dass der Glaukomschaden mit der Größe des Corpus geniculatum laterale [26] und der Abnahme der fraktionalen Anisotropie im Traktus opticus, in der Sehstrahlung und im okzipitalen Kortex [8, 28] korreliert. Ähnliche Befunde fanden sich auch in der diffusionsgewichteten Magnetresonanztomographie (DTI, „diffusion tensor imaging") [7]. Das Neuroimaging zeigt nicht nur die zerebrale Neurodegeneration bei der Glaukomerkrankung, sondern ist auch als Marker für den Schweregrad der Erkrankung hilfreich. Darüber hinaus sind zerebrale Mikroinfarkte als intrazerebraler Risikofaktor für die glaukomatöse Optikusatrophie identifiziert worden [44].

\section{Pathogenese der Glaukom- erkrankung}

Die Pathogenese der Glaukomerkrankung ist multifaktoriell. Das Kennzeichen der Glaukomerkrankung ist der zeitlich beschleunigte Verlust von RGZ. Bei der glaukombedingten Neurodegeneration werden auf molekularer Ebene ähnliche Mechanismen vermutet wie bei chronischen neurodegenerativen

Erkrankungen des zentralen Nervensystems, z.B. der Alzheimer-Erkrankung [45]. Experimentelle Studien an retinalen Zellkulturen und Tiermodellen (chronisch erhöhter IOD, RGZ-Axotomie, Sehnerv-Crush-Modell) weisen darauf hin, dass bei dem glaukomatösen RGZVerlust neben dem Augeninnendruck verschiedene Ursachen wie Exzitotoxizität, oxidativer Stress, gestörter axonaler Transport mit Mangel an neurotrophen Faktoren, mitochondriale Dysfunktion und autoimmune Dysregulation involviert sind [1].

Die Schlüsselhypothesen der Glaukompathogenese umfassen chronisch erhöhten Augeninnendruck [5], Glutamatexzitotoxizität [30], oxidativen Stress [6], Störung des axonalen Transports [10], Neurotrophinmangel [21], mitochondriale Dysfunktion [19], endotheliale Dysfunktion [9], autoimmune Dysregulation [22] und gestörte zentrale InsulinSignalkaskade [11]. Zahlreiche weitere Mechanismen werden diskutiert [12].

\section{Wirkweise von Neuroprotektiva}

Da auch nach IOD-Senkung die Glaukomerkrankung fortschreiten kann, scheinen neuroprotektive Substanzen hilfreich zu sein, und es liegt nahe, die IOD-senkende Therapie durch eine neuroprotektive Therapie zu ergänzen. Neuroprotektive Substanzen zum Erhalt der visuellen Funktion korrigieren das Ungleichgewicht zwischen pro- und antiapoptotischen Signalen und reduzieren bzw. verhindern so den RGZUntergang und Sehbahnschaden.

\section{1) Neuroprotektiva korrigieren das Ungleichgewicht zwischen pro- und antiapoptotischen Signalen}

Dabei machen die bisherigen Ausführungen deutlich, dass bei der Glaukomerkrankung als neurodegenerativer Erkrankung die Therapie mit einer neuroprotektiven Substanz nicht nur auf die retinalen Ganglienzellen, sondern auch auf die im Rahmen der glaukomatösen Dege- neration beteiligten zerebralen Neurone gerichtet sein sollte.

\section{Citicolin}

\section{Indikationen}

Citicolin oder CDP-Cholin, ein nootroper und neurotroper Wirkstoff, welcher in oraler Form seit fast 5 Jahrzehnten in klinischer Anwendung ist und seit einigen Jahren auch als Nahrungsergänzungsmittel in den USA und der EU zur Verfügung steht, erfüllt diese genannte Anforderung zur Prävention und Therapie der glaukomatösen Neurodegeneration. Citicolin eignet sich nach Ansicht der Autoren als ein Neuroprotektivum für alle an der Glaukomerkrankung beteiligten zerebralen Neurone.

Citicolin zeigt auch bei anderen Erkrankungen neuroprotektive Wirkungen. So sind positive Effekt bei der Amblyopie [15] und bei der nichtarteriitischen ischämischen Optikusneuropathie [35] beschrieben. Topisches Citicolin verbessert auch die Funktion und Morphologie der Hornhautnerven bei Diabetes mellitus [14].

\section{Wirkmechanismen}

Citicolin ist eine Vorstufe des Neurotransmitters Acteylcholin sowie anderer neuronaler Membrankomponenten wie Phosphatidylcholin und Sphingomyelin. In verschiedensten experimentellen Ansätzen sind neuroprotektive Eigenschaften von Citicolin nachgewiesen worden. Dabei spielt eine beschleunigte Synthese von Phosphatidylcholin und Phospholipiden eine zentrale Rolle.

Die Neuroprotektion mittels Citicolin erfolgt über glaukomrelevante Mechanismen. Sowohl in Glaukommodellen als auch in Modellen für andere zerebrale neurodegenerative Erkrankungen wirkte Citicolin verschiedenen pathogenetischen Mechanismen entgegen, die auch beim RGZ-Verlust im Rahmen der Glaukomerkrankung eine Rolle spielen. So wirkt Citicolin durch Reduzierung der Glutamat-Exzitotoxizität [29], Reduzierung des oxidativen Stresses [39], Steigerung der Neurotrophinspiegel und Verbesserung des axoplasmatischen Trans- 
Hier steht eine Anzeige.

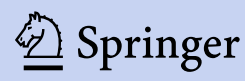


ports [16], Verbesserung der mitochondrialen Funktion [52], Normalisierung der Membranstruktur [51] und Modulation der zerebralen Insulin-Signalkaskade [24] neuroprotektiv.

\section{Klinische Evidenz}

Inzwischen sind 11 klinische Studien publiziert, die alle einen neuroprotektiven Effekt von Citicolin auf die Glaukomerkrankung nachweisen konnten (• Tab. 1). Der erste Bericht über eine Behandlung mit Citicolin bei der Glaukomerkrankung (primäres Offenwinkelglaukom, POWG) mittels i.m.-Citicolin-Injektionen stammt von Pecori-Giraldi et al. aus dem Jahr 1989 [37].

Die Fortführung dieser initialen Studie zeigte einen exzellenten Effekt der Therapie über 10 Jahre: Eine i.m.-Injektion von $1 \mathrm{~g}$ Citicolin täglich für 15 Tage alle 6 Monate führte zu einer signifikanten Stabilisierung des Gesichtsfelds: Bei 2 von 11 der mit Citicolin behandelten vs. 5 von 12 der nicht behandelten Patienten kam es zu einer Gesichtsfeldprogression. Eine Progression war definiert als die Flächenzunahme von Skotomen (SkotomAreal $>500 \mathrm{~mm}^{2}$ ) in der Videoscreen-Perimetrie [48].

Es wurden 10 Jahre nach dieser ersten Studie und ihrer Nachfolgestudie die Ergebnisse einer doppelmaskierten placebokontrollierten Studie publiziert, in der elektrophysiologische Methoden wie VEP und das Muster-Elektroretinogramm (MERG) zur Prüfung der retinalen Funktion und der Sehbahn angewendet wurden. Diese Studie zeigte eine signifikante Verbesserung beider visueller Funktionen nach i.m.-Injektionen von Citicolin [36]. Da die Verbesserungen vorübergehend waren, mussten die Behandlungen alle paar Monate wiederholt werden.

Der positive Effekt von wiederholten Citicolin-Injektionen beim POWG konnte in einer Untersuchung nach einem Verlauf von 8 Jahren durch MERG bestätigt werden [32].

Nun sind i.m.-Injektionen nicht das bevorzugte Therapieregime für eine chronische Erkrankung. Daher wurde in einer kleinen Studie die orale Gabe von Citicolin ( $1 \mathrm{~g}$ pro Tag für 2 Wochen,

Ophthalmologe 2021 · 118:439-448 https://doi.org/10.1007/s00347-021-01362-z

๑) Springer Medizin Verlag GmbH, ein Teil von Springer Nature 2021

\section{A. G. M. Jünemann · P. Grieb · R. Rejdak}

\section{Bedeutung von Citicolin bei der Glaukomerkrankung}

\section{Zusammenfassung}

Hintergrund. In den letzten Jahren wurde in vielen experimentellen und klinischen Studien gezeigt, dass bei der Glaukomerkrankung die neuronale Degeneration nicht nur auf der Höhe von Netzhaut und Sehnerv, sondern im Verlauf der gesamten Sehbahn und des Gehirns erfolgt.

Fragestellung. Dargestellt werden die neuroprotektive Wirkung und ihre Mechanismen von Citicolin bei der Glaukomerkrankung. Material und Methode. Vor dem Hintergrund der Neuroanatomie, des Neuroimaging und der Pathogenese der Glaukomerkrankung wird die Relevanz erläutert. Die Daten der experimentellen und klinischen Studien werden dargelegt und ein Fazit für die klinische Anwendung gezogen.

Ergebnisse. Citicolin wirkt neuroprotektiv über glaukomrelevante Mechanismen. Die neuroprotektive Wirkung von Citicolin bei Offenwinkelglaukomen ist funktionell und morphologisch nachweisbar. Sie ist unabhängig vom Glaukomschaden und
Augeninnendruck und tritt i. d. R. erst nach einem Jahr auf. Die Effekte von oralem Citicolin treten bei einer Tagesdosis von 500-1000 mg auf. Die Einnahme von Citicolin kann dauerhaft oder in Zyklen erfolgen. In den Studien traten bei der Einnahme von Citicolin keine Nebenwirkungen auf. Citicolin kann kognitive Leistungen und somit Therapieadhärenz sowie die Lebensqualität bei Glaukompatienten verbessern

Schlussfolgerung. Diese relativ alte nootrope Substanz, welche jetzt als Nahrungsergänzungsmittel vertrieben wird, scheint eine wertvolle Ergänzung zur konventionellen Therapie der Glaukomerkrankung und eine rationale Option zur Neuroprotektion und Prophylaxe zu sein.

Schlüsselwörter

Nootrope Substanz - Offenwinkelglaukom · Neuroprotektion · Erhöhter Augeninnendruck · Cholin

\section{The role of citicoline in glaucoma}

\section{Abstract}

Background. In recent years, many experimental and clinical studies have shown that in glaucoma, neuronal degeneration occurs not only at the level of the retina and optic nerve, but also along the entire visual pathway and the brain.

Objective. This article presents the neuroprotective effects of citicoline and their mechanisms in glaucoma disease.

Materials and methods. The relevance of citicoline is explained against the background of neuroanatomy, neuroimaging, and the pathogenesis of glaucoma. Data from experimental and clinical studies are presented and a conclusion is drawn for clinical application.

Results. Citicoline has a neuroprotective effect via mechanisms relevant to glaucoma. The neuroprotective effect of citicoline in open-angle glaucoma can be demonstrated functionally and morphologically. It is independent of the glaucoma damage and intraocular pressure, and usually occurs only after 1 year. The effects of oral citicoline occur at a daily dose of 500-1000 mg. Citicoline can be taken permanently or in cycles. No side effects occurred in the studies when taking citicoline. Citicoline can improve cognitive performance and thus treatment adherence as well as quality of life in glaucoma patients. Conclusion. This relatively old nootropic drug, which is now marketed as a food supplement, seems to be a valuable addition to conventional treatment and also a rational option for prophylaxis of open-angle glaucoma.

Keywords Nootropic agents - Open-angle glaucoma Neuroprotection - Ocular hypertension . Choline 


\begin{tabular}{|c|c|c|c|c|c|c|c|c|c|}
\hline Autoren & $\begin{array}{l}\text { Studien- } \\
\text { design }\end{array}$ & Patienten & $\begin{array}{l}\text { Alter (Jahre) } \\
\text { MD (dB) }\end{array}$ & $\begin{array}{l}\text { IODa } \\
(\mathrm{mm} \mathrm{Hg})\end{array}$ & $\begin{array}{l}\text { Appli- } \\
\text { kation }\end{array}$ & $\begin{array}{l}\text { Dosis (pro } \\
\text { Tag) }\end{array}$ & $\begin{array}{l}\text { Therapie- } \\
\text { schema }\end{array}$ & Follow-up & Endpunkte \\
\hline $\begin{array}{l}\text { Pecori- } \\
\text { Giraldi } 1989 \\
\text { [37] }\end{array}$ & Kohortenstudie & $\begin{array}{l}\text { OWG } \\
(n=30 \\
A=47)\end{array}$ & $52,12(25-75)$ & - & i.m. & $1 \mathrm{~g}$ & 10 Tage & 3 Monate & Perimetrie \\
\hline $\begin{array}{l}\text { Parisi } 1999 \\
\text { [36] }\end{array}$ & $\begin{array}{l}\text { Doppelmaskiert, } \\
\text { placebokontrol- } \\
\text { liert }\end{array}$ & $\begin{array}{l}\text { OWG } \\
(n=40) \\
25 \mathrm{C}, 15 \mathrm{~K}\end{array}$ & $\begin{array}{l}45,6 \pm 4,3 \\
-3 \text { bis }-6 \mathrm{~dB}\end{array}$ & $\begin{array}{l}25,10 \pm 1,55 \\
(23-27)\end{array}$ & i.m. & $1 \mathrm{~g}$ & $\begin{array}{l}60 \text { Tage } \\
120 \text { Tage w/o } \\
2 \text { Zyklen }\end{array}$ & 360 Tage & VEP, MERG \\
\hline $\begin{array}{l}\text { Virno } 2000 \\
\text { [48] }\end{array}$ & $\begin{array}{l}\text { Fall-Kontroll- } \\
\text { Studie }\end{array}$ & $\begin{array}{l}\text { OWG } \\
(n=23) \\
11 \mathrm{C}, 12 \mathrm{~K}\end{array}$ & - & - & i.m. & $1 \mathrm{~g}$ & $\begin{array}{l}15 \text { Tage } \\
\text { alle } 6 \text { Monate } \\
20 \text { Zyklen }\end{array}$ & 10 Jahre & Perimetrie \\
\hline $\begin{array}{l}\text { Rejdak } 2003 \\
\text { [40] }\end{array}$ & Kohortenstudie & $\begin{array}{l}\text { OWG } \\
(n=21)\end{array}$ & - & - & p.o. & $1 \mathrm{~g}$ & $\begin{array}{l}14 \text { Tage } \\
2 \text { Tage w/o } \\
2 \text { Zyklen }\end{array}$ & 56 Tage & VEP \\
\hline $\begin{array}{l}\text { Parisi } 2005 \\
{[32]}\end{array}$ & $\begin{array}{l}\text { Doppelmaskiert, } \\
\text { placebokontrol- } \\
\text { liert }\end{array}$ & $\begin{array}{l}\text { OWG } \\
(n=30) \\
15 \mathrm{C}, 15 \mathrm{~K}\end{array}$ & -3 bis $-6 d B$ & $\begin{array}{l}25,10 \pm 1,55 \\
(23-27) \\
17,5 \pm 1,3^{b}\end{array}$ & i.m. & $1 \mathrm{~g}$ & $\begin{array}{l}60 \text { Tage } \\
120 \text { Tage w/o } \\
14 \text { Zyklen }\end{array}$ & 8 Jahre & VEP, MERG \\
\hline $\begin{array}{l}\text { Parisi } 2008 \\
\text { [34] }\end{array}$ & $\begin{array}{l}\text { Fall-Kontroll- } \\
\text { Studie }\end{array}$ & $\begin{array}{l}\text { OWG } \\
(n=60 \\
A=70)\end{array}$ & $\begin{array}{l}52,77 \pm 5,28(38-62) \\
-2 \text { bis }-14 \mathrm{~dB}\end{array}$ & - & $\begin{array}{l}\text { i.m. } \\
\text { oral }\end{array}$ & $\begin{array}{l}1 \mathrm{~g} \\
1,6 \mathrm{~g}\end{array}$ & $\begin{array}{l}60 \text { Tage } \\
120 \text { Tage w/o } \\
2 \text { Zyklen }\end{array}$ & 360 Tage & VEP, MERG \\
\hline $\begin{array}{l}\text { Ottobelli } \\
2013[31]\end{array}$ & Kohortenstudie & $\begin{array}{l}\text { POWG } \\
(n=41)\end{array}$ & $\begin{array}{l}72,5 \pm 11,6 \\
-1,1 \pm 0,7 \mathrm{~dB} / \mathrm{Jahr}^{\mathrm{c}}\end{array}$ & $15,5 \pm 2,6$ & p.o. & $0,5 \mathrm{~g}$ & $\begin{array}{l}4 \times 120 \text { Tage } \\
60 \text { Tage w/o } \\
4 \text { Zyklen }\end{array}$ & 2 Jahre & Perimetrie \\
\hline $\begin{array}{l}\text { Roberti } \\
2014 \text { [41] }\end{array}$ & $\begin{array}{l}\text { Doppelmaskiert, } \\
\text { placebokontrol- } \\
\text { liert }\end{array}$ & $\begin{array}{l}\text { OWG } \\
(n=34) \\
16 \mathrm{C}, 18 \mathrm{~K}\end{array}$ & -3 bis $-12 d B$ & - & AT & $3 \times$ & 60 Tage & 90 Tage & VEP, MERG \\
\hline $\begin{array}{l}\text { Parisi } 2015 \\
\text { [33] }\end{array}$ & $\begin{array}{l}\text { Fall-Kontroll- } \\
\text { Studie }\end{array}$ & $\begin{array}{l}\text { OWG } \\
(n=56) \\
28 \mathrm{C}, 18 \mathrm{~K}\end{array}$ & $\begin{array}{l}52,4 \pm 4,72 \\
(40-60)>-10 \mathrm{~dB}\end{array}$ & $23-28$ & AT & $3 x$ & $\begin{array}{l}120 \text { Tage } \\
60 \text { Tage w/o }\end{array}$ & 180 Tage & VEP, MERG \\
\hline $\begin{array}{l}\text { Lanza } 2009 \\
{[25]}\end{array}$ & $\begin{array}{l}\text { Fall-Kontroll- } \\
\text { Studie }\end{array}$ & $\begin{array}{l}\text { OWG } \\
(n=60) \\
30 \mathrm{C}, 30 \mathrm{~K}\end{array}$ & $\begin{array}{l}\mathrm{C} 64,1 \pm 5,8 \\
\mathrm{~K} 62,9 \pm 7,2 \\
1,0-1,5 \mathrm{~dB} / \mathrm{Jahr} \\
\mathrm{C}-6,51 \pm 2,65 \\
\mathrm{~K}-6,39 \pm 2,03\end{array}$ & $<18$ & p.o. & $0,5 \mathrm{~g}$ & $\begin{array}{l}120 \text { Tage } \\
60 \text { Tage w/o } \\
4 \text { Zyklen }\end{array}$ & 2 Jahre & $\begin{array}{l}\text { Perimetrie, } \\
\text { ОСТ }\end{array}$ \\
\hline $\begin{array}{l}\text { Marino } \\
2020 \text { [27] }\end{array}$ & $\begin{array}{l}\text { Cross-over- } \\
\text { Studie }\end{array}$ & $\begin{array}{l}\text { OWG } \\
(n=109)\end{array}$ & $\begin{array}{l}\text { C } 66,5, \mathrm{~K} 68,0 \\
-1,72 \mathrm{~dB} \\
(-19,0 \text { bis }+3,18 \mathrm{~dB})\end{array}$ & $\begin{array}{l}16 \\
(10-25)\end{array}$ & p.o. & $0,5 \mathrm{~g}$ & $\begin{array}{l}120 \text { Tage } \\
120 \text { Tage w/o }\end{array}$ & 4 Monate & $\begin{array}{l}\text { SPARCS, } \\
\text { GQL-15 }\end{array}$ \\
\hline $\begin{array}{l}\text { Rossetti } \\
2020 \text { [42] }\end{array}$ & $\begin{array}{l}\text { Doppelmaskiert, } \\
\text { placebokontrol- } \\
\text { liert }\end{array}$ & $\begin{array}{l}\text { OWG } \\
(n=80) \\
40 \mathrm{C}, 40 \mathrm{~K}\end{array}$ & $\begin{array}{l}\text { C 74,0, K 71,4 } \\
-9,0 \mathrm{~dB} \\
0,8 \mathrm{~dB} / \mathrm{Jahr}^{\mathrm{C}}\end{array}$ & $\begin{array}{l}\text { C } 14,3 \\
\text { K } 13,8<18^{b}\end{array}$ & AT & $3 x$ & 3 Jahre & 3 Jahre & $\begin{array}{l}\text { Perimetrie, } \\
\text { OCT }\end{array}$ \\
\hline \multicolumn{10}{|c|}{$\begin{array}{l}\text { IOD Augeninnendruck, MD „mean defect“ in statischer Weiß-Weiß-Perimetrie, OWG Offenwinkelglaukom, POWG primäres Offenwinkelglaukom, } A \text { Augen, } \\
\text { C Studiengruppe mit Citicolin-Therapie, K Kontrollgruppe, i.m. intramuskulär, p.o. per oS, oral; AT Augentropfen, w/O „wash-out”, VEP visuell evozierte Poten- } \\
\text { ziale, MERG Muster-Elektroretinogramm, OCT optische Kohärenztomographie, SPARCS Spaeth-Richman-Contrast-Sensitivity-Test, GQL-15 Glaucoma Quality of } \\
\text { Life-15 } \\
\text { 'ODD bei Studieneinschluss } \\
\text { bIm Verlauf der Studienjahre } \\
\text { "Progressionsrate im MD in } 2 \text { Jahren vor Studienbeginn }\end{array}$} \\
\hline
\end{tabular}

2 Wochen Pause, Wiederholung) auf die VEP von POWG-Patienten untersucht [40]. Auch wenn die Studienpopulation klein war (21 Augen von 11 Patienten), zeigte sich eine signifikante Verkürzung der Latenzverlängerung in den VEP und ein signifikanter, aber geringer ausgeprägter Anstieg der VEP-Amplitude.

Dann erfolgten Studien, welche die Wirksamkeit von p.o. und i.m. appliziertem Citicolin verglichen. In einer grö-
Beren italienischen Studie wurde der Effekt von i.m. ( $1 \mathrm{~g} / \mathrm{tgl}$.) und p.o. appliziertem Citicolin $(1,6 \mathrm{~g} / \mathrm{tgl}$.) verglichen. Nach 2 Monaten zeigte sich eine signifikante Verbesserung von MERG und VEP. Dieser Effekt ließ in den folgenden 4 Monaten ohne Therapie wieder nach, jedoch war nach 4 Monaten die Funktion immer noch besser im Vergleich zur Basisuntersuchung. Die Wiederholung der Therapie führte zu einer stufenweisen Verbes- serung [34]. Aus dieser Arbeit schlossen die Autoren, dass Citicolin in der Tat neuroprotektiv, aber nicht kurativ wirkt und eine periodische Therapie notwendig ist, um den Effekt zu erhalten.

In einer weiteren italienischen Studie mit 3 Studienzentren wurde der positive Langzeiteffekt von Citicolin p.o. bestätigt. Dabei wurden 41 Patienten, die ein progredientes POWG trotz eines kontrollierten IOD auswiesen, mit 
$500 \mathrm{mg}$ Citicolin tgl. für 4 Monate mit einem anschließenden therapiefreien Intervall von 2 Monaten behandelt [31]. Die Studie war nicht randomisiert, aber der prospektiven Studienphase ging eine retrospektive Analyse der prätherapeutischen Progressionsrate im Gesichtsfeld voraus. Betrug die Progressionsrate vor Therapie $-1,1 \pm 0,7 \mathrm{~dB} / \mathrm{Jahr}$, so lag sie unter Therapie nach 2 Jahren im Mittel bei $-0,15 \pm 0,3 \mathrm{~dB} / \mathrm{Jahr}$. In einer weiteren Fall-Kontroll-Studie wurde gezeigt, dass es unter einer Therapie mit 500 mg Citicolin p.o. nach 2 Jahren zu einer signifikanten Reduktion der Gesichtsfeldprogression und der Abnahme in der retinalen Nervenfaserschicht in der optischen Kohärenztomographie (OCT) kommt [25].

\section{》) Die Wiederholung der \\ Therapie führte zu einer stufenweisen Verbesserung}

Marino et al. [27] untersuchten die Kontrastempfindlichkeit mit dem SpaethRichman-Contrast-Sensiti-

vity(SPARCS)-Test und die Lebensqualität (Glaucoma Quality of Life-15, GQL15) unter Citicolin-Therapie (500 mg, mit Homotaurin $50 \mathrm{mg}$, Vitamin E $12 \mathrm{mg}$ ) [46] bei 109 Patienten mit primärem Offenwinkelglaukom, dabei betrugen der "mean defect“ in statischer WeißWeiß-Perimetrie, MD, $-1,72 \mathrm{~dB}(-19,0$ bis $+3,18)$, die Glaukomdauer 7 Jahre (1-19) und der IOD $16 \mathrm{~mm} \mathrm{Hg}$ (10-25), $66 \%$ erhielten eine Monotherapie. Jeweils nach 4 Monaten zeigte sich eine signifikante Verbesserung der Kontrastsensitivität und der Lebensqualität.

\section{Applikation}

Neben i.m.- und p.o.-Applikation kann Citicolin auch lokal mittels Augentropfen angewandt werden. Nach topischer Applikation ist Citicolin im Glaskörper nachweisbar [3]. Parisi et al. [33] berichten über den Effekt von topischem Citicolin als Augentropfen (Citicolin 0,2 g, 3 Tropfen tgl.) bei 47 Patienten mit Offenwinkelglaukom (mittleres Alter 52,4 \pm 4,72 Jahre, IOD 23-28 mm Hg ohne Therapie, MD>-10 dB). Alle Patienten wiesen unter einer Monotherapie mit Betablockern eine IOD < $18 \mathrm{~mm} \mathrm{Hg}$ auf. Bei 24 Patienten wurden zusätzlich Citicolin-Augentropfen für 4 Monate mit einer anschließenden Auswaschphase von 2 Monaten angewendet. Nach 4 Monaten zeigten MERG und VEP unter zusätzlicher Citicolin-Gabe zum Betablocker signifikant größere Amplituden und kürzere Gipfellatenzen im Vergleich zur alleinigen Therapie mit Betablockern. Am Ende der 2-monatigen Auswaschphase nach 6 Monaten waren keine Unterschiede mehr nachweisbar. Topisches Citicolin induziert somit eine Verbesserung der bioelektrischen Aktivität in der Netzhaut und im visuellen Kortex.

In einer zweiten randomisierten doppelmaskierten placebokontrollierten multizentrischen Studie über 3 Jahre wurde gezeigt, dass Citicolin zu einer signifikanten Reduktion der funktionellen (Weiß-Weiß-Perimetrie) und morphologischen (Dicke der retinalen Nervenfaserschicht in der OCT) Progression führte [43]. In diese Studie wurden leicht- bis mittelgradige Offenwinkelglaukome eingeschlossen, die bei einem IOD unter $18 \mathrm{~mm} \mathrm{Hg}$ in den 2 Jahren vor Studienbeginn eine Progression in der Perimetrie (MD) von bis $0,5 \mathrm{~dB} / \mathrm{Jahr}$ aufwiesen. Der IOD wurde über die 3 Studienjahre mittels medikamentöser oder chirurgischer Therapie unter $18 \mathrm{~mm} \mathrm{Hg}$ gehalten. Dabei erhielten 80 Patienten Citicolin-Augentropfen $3 \times$ tgl., 80 Patienten Placebo-Augentropfen. Mit Citicolin-Therapie betrug die Gesichtsfeldprogression (MD) nach 3 Jahren $-0,41 \pm 3,45 \mathrm{~dB}$, ohne Citicolin $-2,22 \pm 3,63 \mathrm{~dB}(p=0,02)$. Die Abnahme der retinalen Nervenfaserschichtdicke reduzierte sich im Mittel um $1,13 \mu \mathrm{m}$ ( 1,86 vs. $2,99 \mu \mathrm{m} ; p=0,02)$. Die Studie zeigt, dass eine additive Therapie mit Citicolin zur IOD-senkenden Therapie die Progression bei Patienten mit einer progredienten Glaukomerkrankung trotz IOD $<18 \mathrm{~mm} \mathrm{Hg}$ reduzieren kann [43].

Auch wenn diese Ergebnisse mit den Studienergebnissen bei oraler Applikation vergleichbar waren, so ist die topische Applikation hinsichtlich der Bioverfügbarkeit problematisch. Citicolin ist wasserlöslich und penetriert schlecht die
Cornea. Präklinische Daten haben zwar gezeigt, dass topisches Citicolin den Glaskörper erreichen kann [47]. Die intraokulare Bioverfügbarkeit von Citicolin wurde jedoch nur durch eine benzalkoniumchloridvermittelte Schädigung der Augenoberfläche erreicht [2]. Dies wird wie auch bei der lokalen drucksenkenden Therapie zu einer chronischen Blepharokeratokonjunktivitis führen und ist als Applikationskonzept aus diesen Gründen daher eher nicht sinnvoll. Zudem können die altersbedingten manuellen Einschränkungen die applizierte Dosis beeinflussen. Orales Citicolin hat nach dem bisherigen Wissensstand keine $\mathrm{Ne}$ benwirkungen und ist daher für die Anwendung besser geeignet. Es weist eine gute Bioverfügbarkeit und eine vergleichbare Effektivität wie bei parenteraler Gabe (i.v., i.m.) auf [42].

\section{》) Die topische Applikation ist hinsichtlich der Bioverfügbarkeit problematisch}

Darüber hinaus hat Citicolin nootrope Eigenschaften und wirkt sich somit positiv auf subjektive Gedächtnisstörungen und leicht- bis mittelgradige vaskuläre kognitive Störungen aus [13]. Die begleitende Einnahme von Citicolin mit der IOD-senkenden Therapie kann somit möglicherweise auch einen positiven Effekt auf die Adhärenz zur Glaukomtherapie haben [16].

\section{Citicolin und evidenzbasierte Medizin}

Die Beurteilung von Citicolin angesichts der evidenzbasierten Medizin wirft die Frage auf: Könnte es eine Prophylaxe oder Therapie darstellen? Jain und Aref [20] diskutieren Analogien zwischen seniler Demenz und Glaukom und vermuteten, dass bei beiden Erkrankungen an ein „Neuroenhancement " mittels Substanzen wie Citicolin gedacht werden kann, welches derzeit als Nahrungsergänzungsmittel verfügbar ist.

Auf der anderen Seite stellte das Panel on Dietetic Products, Nutrition and Allergies (NDA) der European Food Safety Authority (EFSA) 2014 hinsichtlich 
Hier steht eine Anzeige.

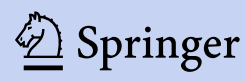


des Wirkstoffs Cytidin-5-Diphosphocholin (CDP-Cholin) im Zusammenhang mit dem Erhalt des normalen Sehens bei älteren Menschen fest, dass zwischen Einnahme von CDP-Cholin und dem Erhalt des normalen Sehens kein $\mathrm{Zu}$ sammenhang im Sinne von Ursache und Wirkung hergestellt werden kann (EFSA NDA 2014).

Diese 2 auf den ersten Blick gegensätzlichen Aussagen zielen auf 2 vollkommen unterschiedliche therapeutische Szenarien ab: die Erste bezieht sich auf die Einnahme von Citicolin bei einer bestehenden (manifesten) Erkrankung, die Zweite bezieht sich jedoch auf die Einnahme von Citicolin durch eine asymptomatische alternde Bevölkerung ohne Erkrankung zur möglichen Prophylaxe einer Erkrankung.

\section{》) Ein Eckstein der EBM ist das hierarchische System der Klassifizierung von Evidenz}

Argumente für eine eher ablehnende Haltung gegenüber oralem Citicolin zum Erhalt einer gesunden Sehkraft im Zusammenhang mit dem Alterungsprozess sind vergleichbar mit denen der evidenzbasierten Medizin (EBM). Evidenzbasierte Medizin ist im Prinzip ein Code für einen Verhaltenskodex, um klinische Entscheidungen zu treffen. Ein Eckstein der EBM ist das hierarchische System der Klassifizierung von Evidenz, bekannt als „Pyramide der Evidenz-Level“, die direkt in den „Empfehlungsgrad“ übersetzt wird [46]. Der Goldstandard für hohe Evidenz sind die Ergebnisse prospektiver randomisierter maskierter sog. Phase-III-Studien mit klar definierten klinischen Endpunkten, ihrer Metaanalysen und systematischen Reviews. Evidenz, die auf nichtrandomisierten klinischen Studien basiert, wird als wesentlich geringer aussagekräftig angesehen, und auf nichtklinischen Studien basierenden Empfehlungen wird eine geringe Aussagekraft zugesprochen.

Ohne Zweifel ist eine abwartende, restriktive Haltung gegenüber Medikamenten sinnvoll, die ein nicht zu vernachlässigendes toxisches Potenzial haben und zugleich teuer sind (typisch für Originalmedikamente). Für die Marktzulassung eines neuen Medikaments muss in einer randomisierten maskierten Studie gezeigt werden, dass der therapeutische Nutzen das potenzielle Risiko der Behandlung überwiegt. Diese Anforderungen sind im Fall von Nahrungsergänzungsmitteln nicht gegeben. Da bei adäquater, richtig dosierter Einnahme Nahrungsergänzung als weitestgehend risikofrei angesehen wird, ist das Thema Effektivität in Relation zum Risiko von untergeordneter Bedeutung. Im Fall von Citicolin wird die tägliche Dosis von $500-1000 \mathrm{mg}$ von der EFSA als vollkommen sicher ohne Nebenwirkungen angesehen.

Es ist auch unrealistisch anzunehmen, dass ein Zusammenhang zwischen der Einnahme eines Nahrungsergänzungsmittels und seinem prophylaktischen bzw. therapeutischen Effekt eindeutig festgestellt werden kann. Als ein Beispiel kann Vitamin C dienen, dessen jährliche Produktion auf $110.000 \mathrm{t}$ geschätzt wird [38]. Trotzdem ist die Effektivität von Vitamin C hinsichtlich der Vorbeugung und Behandlung von Erkältungskrankheiten seit über 70 Jahren Gegenstand kontroverser Diskussionen und bis heute nicht endgültig geklärt [17]. Aktuell gewinnt diese Diskussion im Hinblick auf die Infektion mit dem Coronavirus SARS-CoV-2 („severe acute respiratory syndrome coronavirus 2", CoronaInfektion) oder die Erkrankung daran (COVID-19, „, coronavirus disease 2019“) zusätzlich an Bedeutung [49].

Im Fall der Glaukomerkrankung ist es im Gegensatz zu akuten symptomatischen Erkrankungen schwer, aufgrund des jahrelang asymptomatischen und langsam progredienten Verlaufs sowie des Fehlens von Frühtests bzw. Screening schlüssige klinische Daten zur Effektivität einer Prophylaxe mit einem Nahrungsergänzungsmittel zu gewinnen.

\section{Ausblick}

In einem Standardwerk zum Glaukom von 2010 ist Citicolin nur einmal kurz in einem Kapitel von Zelefski und Ritch [53] zu sog. alternativen und „nicht-traditionellen" freiverkäuflichen Wirkstoffen wie Alpha-Liponsäure, Fischöl, Omega-3-Fettsäuren oder Carnitin erwähnt.
In der Einleitung schreiben die Autoren, dass es aufgrund des Fehlens klinischer Studien zu diesen natürlichen Präparaten der Einschätzung des Lesers überlassen sei, welche effektiv oder nicht effektiv bei der Glaukomerkrankung seien. Die Autoren geben keine eigene Beurteilung ab.

Die Autoren der vorliegenden Arbeit haben aufzuzeigen versucht, dass die Datenlage hinsichtlich der neuroprotektiven Eigenschaften von Citicolin einschließlich klinischer Daten umfangreich und überzeugend ist.

\section{》) In der EU ist Citicolin als Nahrungsergänzungsmittel (2014/423/EU) zugelassen}

Diese relativ alte nootrope Substanz, welche jetzt als Nahrungsergänzungsmittel vertrieben wird, scheint eine wertvolle Ergänzung zur konventionellen Therapie der Glaukomerkrankung und eine rationale Option zur Prophylaxe zu sein. In der EU ist Citicolin als Nahrungsergänzungsmittel (2014/423/EU) zugelassen, in Italien speziell für Patienten mit Glaukomerkrankung. Die derzeitige Datenlage zeigt, dass Citicolin einen neuroprotektiven Effekt bei Offenwinkelglaukomen unabhängig vom Glaukomstadium aufweist. Dieser ist auch bei progredienter Glaukomerkrankung trotz Augeninnendruckwerten unter $18 \mathrm{~mm} \mathrm{Hg}$ nachzuweisen.

\section{Fazit für die Praxis}

- Citicolin wirkt bei Offenwinkelglaukomen neuroprotektiv.

- Die Wirkung von Citicolin ist funktionell und morphologisch nachweisbar.

- Die Neuroprotektion ist unabhängig vom Glaukomschaden und Augeninnendruck.

- Der neuroprotektive Effekt von Citicolin ist $i$. d. R. erst nach einem Jahr nachweisbar.

- Die Effekte von oralem Citicolin treten bei einer Tagesdosis von 500-1000 mg auf.

- Die Einnahme von Citicolin kann dauerhaft oder in Zyklen erfolgen. 


\section{- In den Studien traten bei der Einnah- me von Citicolin keine Nebenwirkun- gen auf. \\ - Citicolin kann kognitive Leistun- gen und somit Therapieadhärenz verbessern. \\ - Citicolin kann die Lebensqualität bei Glaukompatienten verbessern.}

\section{Korrespondenzadresse

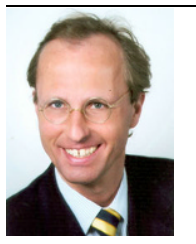 \\ Prof. Dr. med. \\ Anselm G. M. Jünemann, \\ F.E.B.O \\ Department of General and \\ Pediatric Ophthalmology \\ Service, Medical University of Lublin \\ Chmielna 1 str., 20-079 Lublin Polen \\ anselm.juenemann@ \\ outlook.de}

\section{Einhaltung ethischer Richtlinien}

Interessenkonflikt. A.G.M. Jünemann, P. Grieb und R. Rejdak geben an, dass kein Interessenkonflikt besteht.

Für diesen Beitrag wurden von den Autoren keine Studien an Menschen oder Tieren durchgeführt. Für die aufgeführten Studien gelten die jeweils dort angegebenen ethischen Richtlinien.

\section{Literatur}

1. Almasieh M, Wilson AM, Morquette B et al (2012) The molecular basis of retinal ganglion cell death in glaucoma. Prog Retin Eye Res 31:152-181

2. Baudouin C, Labbé A, Liang $\mathrm{H}$ et al (2010) Preservatives in eye drops:the good, the bad and the ugly. Prog Retin Eye Res 29:312-334

3. Carnevale C, Manni G, Roberti G etal (2019) Human vitreous concentrations of citicoline following topical application of citicoline $2 \%$ ophthalmic solution. PLoS ONE 14(2019):e224982. https://doi. org/10.1371/journal.pone.0224982

4. Celiker H, Yuksel N, Solakoglu S et al (2016) Neuroprotective effects of memantine in the retina of glaucomatous rats: an electron microscopic study. J Ophthalmic Vis Res 11:174-182

5. Chan MPY, Broadway DC, Khawaja AP et al (2017) Glaucoma and intraocular pressure in EPIC-Norfolk Eye Study: cross sectional study. BMJ 358:j3889. https://doi.org/10.1136/bmj.j3889

6. Dada T,Mittal D, MohantyKetal (2018)Mindfulness meditation reduces intraocular pressure, lowers stress biomarkers and modulates gene expression in glaucoma: a randomized controlled trial. J Glaucoma 7:1061-1067

7. Dai H, Yin D, Hu C et al (2013) Whole-brain voxelbased analysis of diffusion tensor MRI parameters in patients with primary open angle glaucoma and correlation with clinical glaucoma stage. Neuroradiology 55:233-243
8. Engelhorn T, Michelson G, Waerntges S et al (2012) A new approach to assess intracranial white matter abnormalities in glaucoma patients: changes of fractional anisotropy detected by $3 \mathrm{~T}$ diffusion tensor imaging. Acad Radiol 19:485-488

9. Fadini GP, Pagano C, Baesso l et al (2010) Reduced endothelial progenitor cells and brachial artery flow-mediated dilation as evidence of endothelial dysfunction in ocular hypertension and primary open-angle glaucoma. Acta Ophthalmol 88:135-141

10. Fahy ET, Chrysostomou V, Crowston JG (2016) Impaired axonal transport and glaucoma. Curr Eye Res 41:273-283

11. Faiq MA, Dada T (2017) Diabetes type 4: a paradigm shift in the understanding of glaucoma, the brain specific diabetes and the candidature of insulin as a therapeuticagent. Curr Mol Med 17:46-59

12. Faiq MA, Wollstein G, Schuman JS et al (2019) Cholinergic nervous system and glaucoma: from basic science to clinical applications. Prog Retin Eye Res 72:100767. https://doi.org/10.1016/j. preteyeres.2019.06.003

13. Fioravanti M, Yanagi M (2005) Cytidinediphosphocholine (CDP-choline) for cognitive and behavioural disturbances associated with chronic cerebral disorders in the elderly. Cochrane Database Syst Rev. https://doi.org/10.1002/14651858. CD000269.pub3

14. Fogagnolo $P$, Melardi E, Tranchina L et al (2020) Topical citicoline and vitamin B12 versus placebo in the treatment of diabetes-related corneal nerve damage: a randomized double-blind controlled trial. BMC Ophthalmol 20:315. https://doi.org/10 1186/s12886-020-01584-w

15. Fresina M, Dickmann A, Salerni A et al (2008) Effect of oral CDP-choline on visual function in young amblyopic patients. Graefes Arch Clin Exp Ophthalmol 246:143-150

16. GriebP, Junemann A, Rekas Metal (2016) Citicoline: a food beneficial for patients suffering from or threated with glaucoma. Front Aging Neurosci. https://doi.org/10.3389/fnagi.2016.00073

17. Hemilä H, ChalkerE (2013) Vitamin C for preventing and treating the common cold. Cochrane Database Syst Rev. https://doi.org/10.1002/14651858. CD000980.pub4

18. Horn FK, Bergua A, Jünemann A et al (2000) Visua evoked potentials under luminance contrast and color contrast stimulation in glaucoma diagnosis. J Glaucoma 9:428-437

19. Ito YA, Di Polo A (2017) Mitochondrial dynamics, transport, and quality control: a bottleneck for retinal ganglion cell viability in optic neuropathies. Mitochondrion 36:186-192

20. Jain S, Aref AA (2014) Senile dementia and glaucoma: evidence for a common link. J Ophthalmic Vis Res 10:178-183

21. Johnson TV, BullND, Martin KR(2011) Neurotrophic factor delivery as a protective treatment for glaucoma. Exp Eye Res 93:196-203

22. Jünemann A, Hohberger B, Rech J et al (2018) Agonistic Autoantibodies to the $\beta 2$-Adrenergic receptor involved in the pathogenesis of openangle glaucoma. Front Immunol 9:145. https://doi. org/10.3389/fimmu.2018.00145

23. Jünemann AGM, Huchzermeyer C, Rejdak R (2013) Medikamentöse Glaukomtherapie - Welche Lehren können wir aus den großen klinischen Studien ziehen? Ophthalmologe 110:1134-1148

24. Krupinski J, Abudawood, Matou-Nasri Set al (2012) Citicoline induces angiogenesisimproving survival of vascular/human brain microvessel endothelial cells through pathways involving ERK $1 / 2$ and insulin receptor substrate-1. Vasc Cell 4:20. https:// doi.org/10.1186/2045-824X-4-20

25. Lanza M, Gironi Carnevale UA, Mele L et al (2019) Morphological and functional evaluation of oral citicoline therapy in chronic open-angle glaucoma patients: A pilot study with a 2-year followup. Front Pharmacol 10:1117. https://doi.org/10. 3389/fphar.2019.01117

26. Lee JY, Jeong HJ, Lee JH et al (2014) An investigation of lateral geniculate nucleus volume in patients with primary open-angle glaucoma using 7T magnetic resonance imaging. Invest Ophthalmol Vis Sci 55:3468-3476

27. Marino PF, Rossi GCM, Campagna G et al (2020) Effects of citicoline, homotaurine, and vitamin E on contrast sensitivity and visual-related quality of life in patients with primary open-angle glaucoma: a preliminary study. Molecules 25:5614. https://doi. org/10.3390/molecules25235614

28. Michelson G, Engelhorn T, Wärntges $S$ et al (2013) DTI parameters of axonal integrity and demyelination of the optic radiation correlate with glaucoma indices. Graefes Arch Clin Exp Ophthalmol 251:243-253

29. Mir C, Clotet J, Aledo R et al (2003) CDPcholine prevents glutamate-mediated cell death in cerebellar granule neurons. J Mol Neurosci 20:53-60

30. Osborne NN, Chidlow G, Wood JP (2006) Glutamate excitotoxicity in glaucoma: truth or fiction? By AJ Lotery. Eye 20:1392-1394

31. Ottobelli L, Manni GL, Centofanti M et al (2013) Citicoline oral solution in glaucoma: is there a role in slowing disease progression? Ophthalmologica 229:219-226

32. Parisi V (2005) Electrophysiological assessment of glaucomatous visual dysfunction during treatment with cytidine- $5^{\prime}$-diphosphocholine (citicoline): a study of 8 years of follow-up. Doc Ophthalmol 110:91-102

33. Parisi V, Centofanti M, Ziccardi L et al (2015) Treatment with citicoline eye drops enhances retinal function and neural conduction along the visual pathways in open angle glaucoma. Graefes Arch Clin Exp Ophthalmol 253:1327-1340

34. Parisi V, Coppola G, Centofanti M et al (2008) Evidence of the neuroprotective role of citicoline in glaucoma patients. Prog Brain Res 173:541-554

35. Parisi V, Coppola G, Ziccardi L et al (2008) Cytidine5 -diphosphocholine (citicoline): a pilot study in patients with nonarteritic ischaemic optic neuropathy. Eur J Neurol 15:465-474

36. Parisi V, Manni G, Colacino G et al (1999) Cytidine5 -diphosphocholine (citicoline) improves retinal and cortical responses in patients with glaucoma. Ophthalmology 106:1126-1134

37. Pecori-Giraldi J, Virno M, Covelli G et al (1989) Therapeutic value of citicoline in the treatment of glaucoma (computerized and automated perimetric investigation). Int Ophthalmol 13:109-112

38. PollakP (2011) Fine chemicals: the industry and the business, 2. Aufl. Willey, Hoboken

39. Qian K, Gu Y, Zhao Y et al (2014) Citicoline protects brain against closed head injury in rats through suppressing oxidative stress and calpain overactivation. Neurochem Res 39:1206-1218

40. Rejdak R, Toczołowski J, Kurkowski J et al (2003) Oral citicoline treatment improves visual pathway function in glaucoma. Med Sci Monit 9:PI24-PI28

41. RobertiG, Tanga L,Parisi Vetal (2014) A preliminary study of the neuroprotective role of Citicoline eye drops in glaucomatous optic neuropathy. Indian J Ophthalmol 62:549-553 
42. Roda A, Fini A, Grigolo B et al (1983) Routes of administration and serum levels of [Methyl14C]-Cytidine Diphosphocholine. Curr Ther Res 34:1049-1053

43. Rossetti $L$, lester $M$, Tranchina $L$ et al (2020) Can treatment with citicoline eyedrops reduce progression in glaucoma? The results of a randomized placebo-controlled clinical trial. J Glaucoma 29:513-520

44. Schoemann J, Engelhorn T, Waerntges Setal (2014) Cerebral microinfarcts in primary open-angle glaucoma correlated with DTI-derived integrity of optic radiation. Invest Ophthalmol Vis Sci 55:7241-7247

45. Sen S, Saxena R, Tripathi M et al (2020) Neurodegeneration in Alzheimer's disease and glaucoma: overlaps and missing links. Eye 34:1546-1553

46. Shekelle PG, Woolf SH, Eccles M et al (1999) Developing clinical guidelines. West J Med 170:348-351

47. Sun Y, Erdem E, Lyu A et al (2016) The SPARCS: A novel assessment of contrast sensitivity and its reliability in patients with corrected refractive error. Br JOphthalmol 100:1421-1426

48. Virno M, Pecori-Giraldi J, Liguori A et al (2000) The protective effect of citicoline on the progression of the perimetric defects in glaucomatous patients (perimetric study with a 10-year follow-up). Acta Ophthalmol Scand Suppl 232:56-57

49. Wehrmann M (2020) Vitamin C zum Schutz vor SARS-CoV-2 und zur Behandlung von COVID-19. J Gynäkol Endokrinol. https://doi.org/10.1007/ s41975-020-00155-y

50. Weinreb RN, Liebmann JM, Cioffi GA et al (2018) Oral memantine for the treatment of glaucoma: design and results of 2 randomized, placebocontrolled, phase 3 studies. Ophthalmology 125:1874-1885

51. Yildirim T, Eylen A, Lule S et al (2015) Poloxamer188 and citicoline provide neuronal membrane integrityand protectmembrane stability in cortical spreading depression. Int J Neurosci 125:941-946

52. Zazueta C, Buelna-Chontal M, Macias-Lopez A et al (2018) Cytidine- $5^{\prime}$-Diphosphocholine protects the liver from ischemia/reperfusion injury preserving mitochondrial function and reducing oxidative stress. Liver Transpl 24:1070-1083

53. Zelefsky JR, Ritch R (2010) Alternative and non-traditional treatments of glaucoma. In: Schacknow PN, Samples JR (Hrsg) The Glaucoma Book. A Practical, Evidence-Based Approach to Patient Care. Springer, NewYork, S 657-669 (Chapter 56)

54. http://www.who.int/blindness/causes/en/

\section{In eigener Sache}

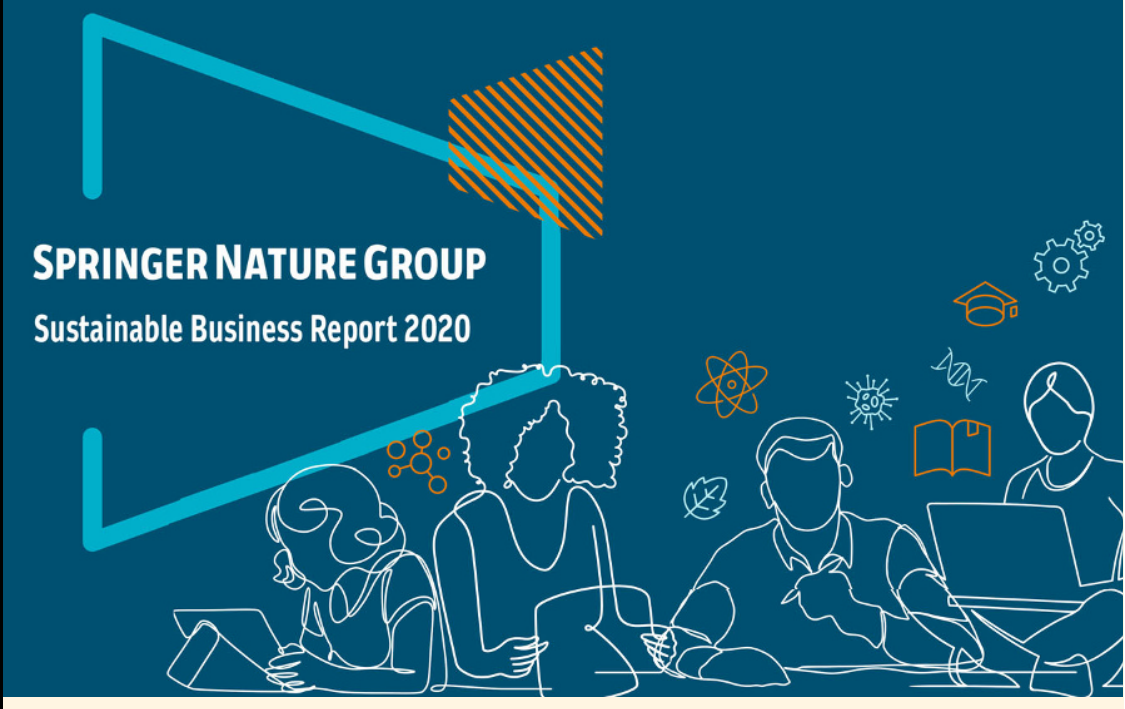

\section{Nachhaltigkeit bei Springer Nature}

Unser Sustainable Business Report 2020 zeigt, welche Fortschritte wir in den Bereichen Umwelt, Soziales und Unternehmensführung über die letzten Jahre erzielen konnten und berücksichtigt dabei auch externe Einflüsse wie die außergewöhnliche Situation im Jahr 2020 durch die COVID-19Pandemie. Als Unternehmen, das nachhaltig und verantwortungsvoll denkt und handelt wollen wir einen positiven Beitrag leisten. Verantwortung ist einer unserer Grundwerte und wir sind bestrebt, als nachhaltiges und ethisches Unternehmen zu handeln, das sich verpflichtet, die Auswirkungen seiner Tätigkeit auf die Umwelt zu berücksichtigen. Wir sind überzeugt, dass die von uns veröffentlichten Inhalte wichtige Gespräche und Diskussionen ermöglichen, die sich positiv auf Gesellschaft und Umwelt auswirken und wollen dieses Potential weiter ausbauen.

Im März 2020 haben wir uns dazu verpflichtet, klimaneutral zu werden. Bereits Ende 2020 haben wir dieses Ziel erreicht und sind - im Hinblick auf unsere Büroräumlichkeiten, Fahrzeugflotte und Geschäftsreisen - klimaneutral. Möglich wurde dies durch die erheblich gesteigerte Nutzung von Ökostrom und CO2-Ausgleichszahlungen. Als Verlag, der aktuellste Forschungsergebnisse zum Klimawandel veröffentlicht, wollen wir außerdem die Aufmerksamkeit für dieses Thema stärken - extern, aber auch intern bei rund $10.000 \mathrm{Be}$ schäftigten, die weltweit bei Springer Nature arbeiten.
Durch den Einsatz neuer Technologien wurden 2020 mehr als drei Milliarden Artikel und Buchkapitel über unsere Plattformen heruntergeladen und damit 50\% mehr als 2019. Für uns ist Technologie der Schlüssel, um Wissen schneller, nachhaltiger und kostengünstiger für noch mehr Menschen in aller Welt verfügbar zu machen.

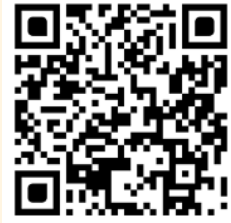

Mit dem QR-Code zur Kurzzusammenfassung des Nachhaltigkeitsreport.

Unsere Veröffentlichung im Jahr 2020:

- 3.000+ Zeitschriften

- 372.000+ Forschungsartikel

- 13.000+ Neue Bücher

- 100 Neue Lehrbuchreihen, die von mehr als 30 Millionen Studierenden genutzt werden 